\title{
A network landscape model: stability analysis and numerical tests
}

\author{
E. Bonacinia ${ }^{\mathrm{a}}$, M. Groppi ${ }^{\mathrm{a}, *}$, R. Monaco ${ }^{\mathrm{b}}$, A. J. Soares ${ }^{\mathrm{c}}$, C. Soresina $^{\mathrm{d}}$ \\ ${ }^{a}$ Dipartimento di Matematica e Informatica, Università di Parma, \\ Parco Area delle Scienze 53/A, 43124 Parma, Italy \\ ${ }^{b}$ DIST, Politecnico di Torino, Viale Mattioli 39, 10125 Torino, Italy \\ ${ }^{c}$ CMAT, Universidade do Minho, Campus de Gualtar, 4710-057 Braga, Portugal \\ ${ }^{d}$ Dipartimento di Matematica "F. Enriques", Università di Milano, \\ via Cesare Saldini 50, 20133 Milano, Italy
}

\begin{abstract}
A Network Landscape Model (NLM) for the evaluation of the ecological trend of an environmental system is here presented and investigated. The model consists in a network of dynamical systems, where each node represents a single Landscape Unit (LU), endowed by a system of ODEs for two variables relevant to the production of bio-energy and to the percentage of green areas, respectively. The main goal of the paper consists in testing the relevance of connectivity between the LUs. For this purpose we consider first the Single LU Model (SLM) and investigate its equilibria and their stability, in terms of two bifurcation parameters. Then the network dynamics is theoretically investigated by means of a bifurcation analysis of a proper simplified differential system, that allows to understand how the coupling between different LUs modifies the asymptotic scenarios for the single LU model. Numerical simulations of NLM are performed, with reference to an environmental system in Northern Italy, and results are discussed in connection with SLM.
\end{abstract}

Keywords: Landscape ecology, Ecological networks, Stability analysis, Bifurcations

\section{Introduction}

The problem of understanding the governing principles of ecosystems can be even found in a paper by Lotka [1] in 1922. But it is at the beginning of this century that the problem of a quantitative evaluation of an environmental system has been posed within the so-called discipline of Landscape Ecology. In fact, the European Landscape Convention of 2000 has encouraged all the European countries to define their landscape objectives on the ground of management and planning of territory through government, conservation and protection of landscapes. On this subject it is now possible to find a large bibliography (see amongst others $[2,3,4]$ and the bibliography therein).

Landscape Ecology may be considered an interdisciplinary field of research involving at the same time empirical testing and mathematical modeling. In this context an environmental system [5] is considered as a spatially extended heterogeneous system that can

\footnotetext{
* Corresponding author

Email addresses: elena.bonacini@unife.it (E. Bonacini), maria.groppi@unipr.it (M. Groppi), roberto.monaco@polito.it (R. Monaco), ajsoares@math.uminho.pt (A. J. Soares), cinzia.soresina@unimi.it (C. Soresina)
} 
be distributed in several Landscape Units (LU) identified by natural or anthropic barriers (roads, speedways, railways, building, industrial infrastructures, rivers, hill ridges, ...) exchanging flows of materials and bio-energy. Moreover, each LU is formed by different biotopes, each characterized by an uniform land cover of vegetation. From a quantitative point of view each LU, often called ecological sector, is characterized by the production of bio-energy and by its capability of transmitting such an energy to the neighboring sectors. Such a characterization of an environmental system has been considered in the book [6] where a quantitative evaluation of its ecological state has been proposed by the so-called ecological graph which, through the computation of suitable indicators (see [7]) determinable by the Geographic Information System (GIS) [8], fixes the values of bio-energy production and flux to the neighbors. On the other hand, the ecological graph may be considered a static picture of the ecological state of the system, whereas natural ecosystems stand in a meta-stable equilibrium which can be modified later in time in a significant way under strong perturbations due to human land uses impact or to natural events $[9,10]$. For this reason, simulation by mathematical models may be an useful and reliable tool for the information about environment trend towards possible future scenarios, presenting even bifurcation phenomena, for some critical values of environmental indicators $[11,12]$.

A first attempt in this direction has been proposed in papers $[13,14]$ where a mathematical model represented by a set of ODEs has been derived. In particular, the main idea of these papers, as well as that of the present one, consists in considering that the equilibrium solutions of the equations correspond to possible different scenarios reachable by the environmental system under investigation. Moreover, the stability analysis derived in [14] has pointed out that bifurcations may arise for some critical values of the parameters, as for instance for the connectivity index which takes into account the level of bio-energy exchanges between the various LUs present in the territory.

More in details, paper [13] assumes as state variables, for the whole environmental system, a generalized form of bio-energy (indicated with the symbol $M$ ) and the total surface of green areas (indicated with $V$ ) presenting high ecological quality. In order to obtain a more detailed description of the ecological state of the environment, in the last part of paper [14] another model is proposed where the state variables (the same of paper [13]) are defined at the level of each LU. In these papers the connectivity index (considered as the control parameter of the dynamical system) is included in the model as a coefficient of the equations and the variable $M$ is defined as a product of the so-called Biological Territorial Capacity (BTC) by a parameter accounting for some morphological and physical property of the biotopes belonging to each LU.

In the present paper we propose a new model called Network Landscape Model (NLM) where the state variables are again defined for each LU. This model differs substantially from that of paper [14]. First of all, the state variable $M$ is replaced by the BTC of each LU (indicated with $B$ ), the other state variable being $V$, the high ecological quality areas of each LU. Such a choice, in our opinion, seems to be reasonable since BTC, contrary to $M$, is strictly related to bio-energy and measurable. The second difference is that the morphological and physical properties of biotopes are included in the model as coefficients of the equations and not as factors of the state variable $M$. But the main novelty consists in adding to the model equations a new term accounting for connectivity, which represents the coupling between the LUs. Such a term is borrowed from electrical synapses linking neurons, more specifically from the so called electrically coupling networks [15]. These novelties have been introduced with the aim of a better calibration of the model, suggested 
by the study cases presented in paper [14] and in the master degree thesis [16].

The paper is organized as follows. In the next section we present the Network Landscape Model as a network of dynamical systems, each of them having the same qualitative structure, that we call Single LU Model (SLM); the last model represents the case of an environmental system where the LUs are not connected and are completely isolated from each other. In Section 3 we perform a stability analysis of the SLM in terms of two bifurcation parameters, detecting general conditions for the number of equilibria, for their existence and stability. In Section 4 we consider the network of LUs, composed by $n$ LUs coupled through a diffusive term proportional to the difference between the bio-energies of each LU. We investigate the asymptotic behaviors of the network (in terms of equilibria and their stability) by means of a proper simplified system, whose dynamics is completely determined in terms of two additional bifurcation parameters. Then, in Section 5, we show some numerical tests relevant to a network of LUs in an environmental system of the northern side of the Turin Province (Italy), characterized by five LUs where rather compact built-up territorial patches interact with natural reserve areas. The results are compared with those of the simplified system and of the single LU model, in order to underline how the coupling between the LUs may modify the scenarios, thanks to the exchange of bio-energy, and how the simplified system is able to give information about the asymptotic behaviour of the network model, in accordance with the stability analysis performed. Some concluding remarks are reported in Section 6.

\section{The network landscape model}

According to the Introduction and to the previous papers $[13,14]$, the environmental system is described as a landscape of $n$ LUs. Then it is possible to represent it as a network of dynamical systems, composed by $n$ nodes, each of them described by a vector state variables $\boldsymbol{x}_{i}(t) \in \mathbb{R}^{2}, i=1, \ldots, n$, interacting pairwise through a set of link, that encode the network topology. We suppose that each LU, when isolated from the others, has its own dynamics described by a system of two differential equations of type $\boldsymbol{x}_{i}^{\prime}=\boldsymbol{F}_{i}\left(\boldsymbol{x}_{i}\right)$. Considering the network landscape, the dynamics of each LU is affected by the neighboring LUs; this can be modeled by adding an interaction term in the single LU model. Then, the evolution of the whole network can be modeled by a system of $2 n$ differential equations, given by

$$
\left\{\begin{array}{l}
\boldsymbol{x}_{i}^{\prime}=\boldsymbol{F}_{i}\left(\boldsymbol{x}_{i}\right)+\boldsymbol{H}_{i}\left(\boldsymbol{x}_{1}, \ldots, \boldsymbol{x}_{n}\right) \\
i=1, \ldots, n
\end{array}\right.
$$

where the function $\boldsymbol{F}_{i}$ describes the dynamics of the $i$-th $\mathrm{LU}$ in the same way as in the single landscape model, whereas the term $\boldsymbol{H}_{i}$ describes the interaction of the $i$-th LU with the other LUs, and, thus, $\boldsymbol{H}_{i}$ depends on all LUs state variables. In order to complete the network landscape model, we have to specify the functions $\boldsymbol{F}_{i}$ and the interaction terms $\boldsymbol{H}_{i}$.

Let us start by describing the single node dynamics $\boldsymbol{F}_{i}$. Each LU is formed by $m_{i}$ biotopes, $i=1, \ldots n$. Each biotope is characterized by its bio-energy or more precisely by its BTC index [2]. Such an index will be indicated in what follows by $B_{j i}, j=1, \ldots, m_{i}$, and assumes values in the range $\left[0, B_{\max }\right]$, with $B_{\max }=6.5 \mathrm{Mcal} /\left(\mathrm{m}^{2} \cdot\right.$ year $)$. The BTC is a synthetic function which takes into account ecosystem metabolism through biomass information, gross primary production and respiration (information about the BTC index 
values for different types of vegetation can be found in $[2,13])$. Moreover, the biotopes having a vegetation with a BTC index greater than 2.5 will be considered hereinafter those of high ecological quality. The total value of BTC of each LU in Mcal/year is given by

$$
B_{i}(t)=\sum_{j=1}^{m_{i}} B_{j i} s_{j i}
$$

where $s_{j i}$ is the area of the $j$-th biotope belonging to the $i$-th LU of total area $S_{i}=\sum_{j=1}^{m_{i}} s_{j i}$.

Let us assume as state variables the total BTC $B_{i}$ of each LU and the sum $V_{i}$ of all the areas of the biotopes of high ecological quality. Then the equations of the SLM for the $i$-th LU read as

$$
\begin{gathered}
B_{i}^{\prime}(t)=a_{i} B_{i}(t)\left(1-\frac{B_{i}(t)}{B_{i}^{\text {max }}}\right)-\ell_{i}\left(1-\frac{V_{i}(t)}{S_{i}}\right) B_{i}(t) \\
V_{i}^{\prime}(t)=d_{i} V_{i}(t)\left(1-\frac{V_{i}(t)}{S_{i}}\right) \frac{B_{i}(t)}{B_{i}^{\text {max }}}-h_{i} U_{i} V_{i}(t)
\end{gathered}
$$

where

$$
B_{i}^{\max }=B_{\max } S_{i}
$$

is the value of the BTC produced by a LU having all the biotopes with a BTC index equal to $B_{\max }$.

Equations (2) and (3) have the same mathematical structure of those proposed in [14] but with coefficients having a different meaning, as already discussed in the Introduction. In fact, the parameters $\ell_{i} \in[0,1]$ are here defined as the ratio between the areas of the impermeable barriers present in the LUs and $S_{i}$, and the parameters $a_{i}$ take into account the capability of the $i$-th LU to produce an increment of bio-energy. Such parameters $a_{i}$ are assumed to depend essentially on the solar exposure of the biotopes, and can be computed by a formula proposed in paper [13] and reported here in the Appendix A at the end of the paper.

Another novelty relies on the coefficients $d_{i}$ of Eqs. (3) which are here considered as dependent on solar exposure, relative humidity and ecotonal length (this last quantity is the length of the borders between biotopes). In fact, it is reasonable that the increasing of green areas of high ecological quality depends on the bio-energy produced in the LU, taking into account as well particular features of the biotopes. Again, the formulas that allow to compute the coefficients $d_{i}$ can be found in the Appendix A.

The last two parameters $h_{i}$ and $U_{i}$, already considered in paper [14] and appearing in Eq. (3), take into account the presence of built-up areas inside the LU that causes impact to the flow of bio-energy. The parameter $h_{i}$ is given by the ratio between the sum of the perimeters of the built-up areas and the total perimeter of the LU, whereas $U_{i}$ is defined as the ratio between the sum of the built-up areas and the total area $S_{i}$ of the LU. Therefore, these parameters can be considered as a measure of the dispersion and of the intensity of constructions inside the LU, respectively. According to its definition, the parameter $h_{i}$ can assume values greater than one (such values mean that construction dispersion in the $\mathrm{LU}$ is significantly remarkable); conversely $U_{i}$ ranges in $[0,1]$.

It is convenient to normalize Eqs. (2)-(3) by dividing the former by $B_{i}^{\max }$ and the latter by $S_{i}$. By introducing the normalized variables $b_{i}=B_{i} / B_{i}^{\max }$ and $v_{i}=V_{i} / S_{i}$, the 
equations for the $i$-th LU result in:

$$
\left\{\begin{array}{l}
b_{i}^{\prime}(t)=F_{i}^{(1)}\left(b_{i}, v_{i}\right)=a_{i} b_{i}(t)\left[1-b_{i}(t)\right]-\ell_{i}\left[1-v_{i}(t)\right] b_{i}(t) \\
v_{i}^{\prime}(t)=F_{i}^{(2)}\left(b_{i}, v_{i}\right)=d_{i} v_{i}(t)\left[1-v_{i}(t)\right] b_{i}(t)-h_{i} U_{i} v_{i}(t) .
\end{array}\right.
$$

Equations (4) define the Single Landscape Model (SLM) proposed here. Throughout the paper, we will refer to them as SLM equations. They have the form $\boldsymbol{x}_{i}^{\prime}=\boldsymbol{F}_{i}\left(\boldsymbol{x}_{i}\right)$ with $\boldsymbol{x}_{i}=\left(b_{i}(t), v_{i}(t)\right)^{T}$.

Let us now define the interaction term $\boldsymbol{H}_{i}$ in (1). The coupling between the $i$-th LU and its $k$ neighbors can be modeled by a linear interaction term proportional to the difference between the bio-energies of the $i$-th and $k$-th LU, affecting only the equation for the bio-energies themselves. Therefore

$$
\boldsymbol{H}_{i}\left(\boldsymbol{x}_{1}, \boldsymbol{x}_{2}, \ldots, \boldsymbol{x}_{n}\right)=\left(\begin{array}{c}
\sum_{k \in \mathcal{I}_{i}} c_{k i}\left[b_{k}(t)-b_{i}(t)\right] \\
0
\end{array}\right)
$$

where the set $\mathcal{I}_{i}$ collects all the indices of the LUs neighboring to the $i$-th one. The term $\boldsymbol{H}_{i}$ has the same mathematical structure as the electric coupling in neural networks [15]. Summing up, we can then write the $2 n$ equations of the network of LUs as

$$
\left\{\begin{array}{l}
b_{i}^{\prime}(t)=a_{i} b_{i}(t)\left[1-b_{i}(t)\right]-\ell_{i}\left[1-v_{i}(t)\right] b_{i}(t)+\sum_{k \in \mathcal{I}_{i}} c_{k i}\left[b_{k}(t)-b_{i}(t)\right] \\
v_{i}^{\prime}(t)=d_{i} v_{i}(t)\left[1-v_{i}(t)\right] b_{i}(t)-h_{i} U_{i} v_{i}(t) \\
i=1, \ldots n
\end{array}\right.
$$

Equations (5) define the Network Landscape Model (NLM) proposed in this paper, we will refer to them as NLM equations.

The coefficients $c_{k i}$ in the expression of $\boldsymbol{H}_{i}$ are the connectivity indices between the $k$-th and the $i$-th LUs and can be computed (see [14]) by the formula

$$
c_{k i}=\frac{H_{k i}}{L_{k i}}, \quad H_{k i}=\sum_{r=1}^{s} L_{k i}^{r} p^{r}, \quad L_{k i}=\sum_{r=1}^{s} L_{k i}^{r},
$$

where $L_{k i}$ is the length of the border between the two LUs, which is divided into $s$ parts, each of length $L_{k i}^{r}$ with a permeability index $p^{r} \in[0,1]$, with 0 for impermeable and 1 for completely permeable.

In order to study the dynamics of the NLM, Eqs. (5) must be equipped with the initial data

$$
b_{i}(0)=b_{i 0}, \quad v_{i}(0)=v_{i 0}
$$

that can be obtained directly from the GIS of the territory under investigation.

\section{Equilibria and stability properties: the case of a single LU}

In this section we investigate the single node dynamics by studying the equilibria of the SLM equations (4) and their stability. In order to simplify the notation, we omit the LU index $i$. 
First of all, we notice that the square $Q=[0,1] \times[0,1]$ in the $(b, v)$ plane is an invariant region for the SLM equations (4) and this guarantees the consistency of the model with the assumption that the normalized variables $b, v$ are meaningful only if they range between 0 and 1. An important property of the SLM (4) is its cooperative structure [17], namely it can be put in the form $\boldsymbol{x}^{\prime}=\boldsymbol{F}(\boldsymbol{x})$, where $\boldsymbol{x}=(b, v)$ and $\boldsymbol{F}$ is a cooperative vector field, meaning that $\partial F^{(k)} / \partial x_{j} \geq 0$ for $k=1,2$ and $j \neq k$. As a consequence, since the dynamics is confined in the compact set $Q$, the long time behavior is severely limited: there will be at least one stable equilibrium, there are no periodic orbits and trajectories will always converge (eventually monotonically) to a stable equilibrium $[17,19]$.

By setting the right hand sides of the SLM equations (4) equal to zero, we compute the equilibrium solutions of the model. Simple calculations lead to four equilibria given by

$$
\begin{gathered}
E_{0}=(0,0), \quad E_{1}=\left(b_{1}, 0\right)=(1-\alpha, 0), \\
E_{*}^{ \pm}=\left(b_{*}^{ \pm}, v_{*}^{ \pm}\right)=\left(b_{*}^{ \pm}, 1-\frac{r}{b_{*}^{ \pm}}\right)=\left(\frac{1 \pm \sqrt{1-4 \alpha r}}{2}, 1-\frac{1 \mp \sqrt{1-4 \alpha r}}{2 \alpha}\right),
\end{gathered}
$$

where

$$
\alpha=\ell / a, \quad r=h U / d .
$$

Let us comment that the equilibrium $E_{0}$ corresponds to a scenario where the system tends to gradually loose its ecological quality and presents a strong landscape fragmentation. The second equilibrium $E_{1}$ represents a scenario with no high quality vegetation, but with some production of bio-energy: such scenarios are typical of territories where agricultural production is predominant. Finally, the coexistence equilibria $E_{*}^{ \pm}$show a good level of bio-energy production in presence of a certain amount of high ecological quality (green) areas.

Since $b$ and $v$ are normalized variables, these equilibria are significant only if their components are between 0 and 1 . In particular, it is obvious to see that the first equilibrium $E_{0}$ is admissible for every choice of the parameters, while $E_{1}$ lies in $Q$ if and only if $\alpha<1$, namely low presence of impermeable barriers (small values of $\ell$ ), together with a good solar exposure (large values of $a$ ). The admissibility of the coexistence equilibria $E_{*}^{ \pm}$depends on both $r$ and $\alpha$ (see Fig. 1); standard calculations allow us to prove that

$$
E_{*}^{+} \in Q \text { iff } r \leq\left\{\begin{array} { l l } 
{ 1 - \alpha } & { \text { when } \alpha < 1 / 2 } \\
{ 1 / ( 4 \alpha ) } & { \text { when } \alpha \geq 1 / 2 }
\end{array} ; \quad E _ { * } ^ { - } \in Q \text { iff } \left\{\begin{array}{l}
\alpha \geq 1 / 2 \\
1-\alpha \leq r \leq 1 /(4 \alpha)
\end{array}\right.\right.
$$

Remark 1. The ecological meaning of the parameters allows us to characterize the quality of a territory in terms of $\alpha$ and $r$. More precisely, low presence of constructions (small values of $h$ and $U$ ) together with good environmental parameters (large values of $d$ ) yield small value of the parameter $r$; low presence of impermeable barriers (low values of $\ell$ ) and high capability to increment the bio-energy (high values of $a$ ) lead to small values of parameter $\alpha$. Therefore, we expect small values of $\alpha$ and $r$ to be related to fertile areas, while high values of both parameters represent highly built-up areas or desert zones. Indeed, for high values of $\alpha$ and $r$ the only feasible equilibrium is $E_{0}$ that will be globally attractive, owing to the cooperative structure of the SLM (4).

The coexistence equilibria $E_{*}^{ \pm}=\left(b_{*}^{ \pm}, v_{*}^{ \pm}\right)$are related to territories with a certain amount of high ecological quality areas and a good production of bio-energy, while $E_{1}=\left(b_{1}, 0\right)$ 
represents lower quality territories with predominance of agricultural production. By simple calculations, it is possible to show that the corresponding equilibrium values $b_{*}^{ \pm}$of the bio-energy are always greater than the equilibrium value $b_{1}$, in accordance with the ecological meaning of the equilibria.

The coexistence equilibria $E_{*}^{ \pm}$are admissible when $r$ is sufficiently small, with threshold decreasing with $\alpha$; in other words, unfavorable parameters $\ell$ and $a$ must be balanced by proper favorable values of $h, U$ and $d$ to get the coexistence of the high ecological quality areas and a good production of bio-energy. In addition, when $\alpha$ and $r$ are both small enough (highest quality territory) the equilibrium state $E_{*}^{-}$, less valuable than $E_{*}^{+}$, is not present.

\section{Proposition 1.}

(i) $E_{0}$ is locally asymptotically stable if and only if $\alpha>1$;

(ii) $E_{1}$ is locally asymptotically stable if and only if $r>1-\alpha$;

(iii) $E_{*}^{+}$is locally asymptotically stable, while $E_{*}^{-}$is a saddle (when they are admissible).

Proof. In order to study the local stability of the equilibria we evaluate the Jacobian matrix of SLM equations (4)

$$
J=\left(\begin{array}{cc}
a[(1-2 b)-\alpha(1-v)] & a \alpha b \\
d v(1-v) & d[b(1-2 v)-r]
\end{array}\right)
$$

at each equilibrium.

(i) It is easy to see that $J\left(E_{0}\right)$ is a diagonal matrix, whose eigenvalues are $\lambda_{1}=a(1-\alpha)$ and $\lambda_{2}=-r d$, and then both are negative iff $\alpha>1$.

(ii) The Jacobian matrix evaluated at $E_{1}$ is an upper triangular matrix, whose eigenvalues are $\lambda_{1}=a(\alpha-1)$ and $\lambda_{2}=d(1-\alpha-r)$. The first eigenvalue is always negative when $E_{1}$ is admissible (i.e. $\alpha<1$ ), while the second one is negative if and only if $r>1-\alpha$.

(iii) The Jacobian matrix evaluated at the coexistence equilibria can be written in the compact form

$$
J\left(E_{*}^{ \pm}\right)=\left(\begin{array}{cc}
-a b_{*}^{ \pm} & a \alpha b_{*}^{ \pm} \\
d v_{*}^{ \pm}\left(1-v_{*}^{ \pm}\right) & -d b_{*}^{ \pm} v_{*}^{ \pm}
\end{array}\right)
$$

The determinant of $J\left(E_{*}^{ \pm}\right)$, by simple calculations, results:

$$
\operatorname{det}\left(J\left(E_{*}^{ \pm}\right)\right)=a d v_{*}^{ \pm} b_{*}^{ \pm}\left(b_{*}^{ \pm}-\alpha\left(1-v_{*}^{ \pm}\right)\right)= \pm a d v_{*}^{ \pm} b_{*}^{ \pm} \sqrt{1-4 \alpha r} .
$$

We notice that the square root is well defined, thanks to the admissibility conditions (7) for the coexistence equilibria since $1-\alpha<1 /(4 \alpha)$ for $\alpha<1 / 2$. The determinant of $J\left(E_{*}^{-}\right)$turns out to be always negative and therefore $E_{*}^{-}$is a saddle point, whenever it is admissible. The determinant of $J\left(E_{*}^{+}\right)$is instead always positive. As regards the sign of the trace of $J\left(E_{*}^{ \pm}\right)$, the diagonal elements of the matrix (8) are both negative, therefore $\operatorname{tr} J\left(E_{*}^{+}\right)<0$.

The results about existence and stability of equilibrium states are summarized in Fig. 1. In the parameter space $(\alpha, r)$ the lines $r=1-\alpha, \alpha=1$ and the hyperbola $r=1 /(4 \alpha)$ divide the first quadrant in five regions, which are qualitatively different either for the 


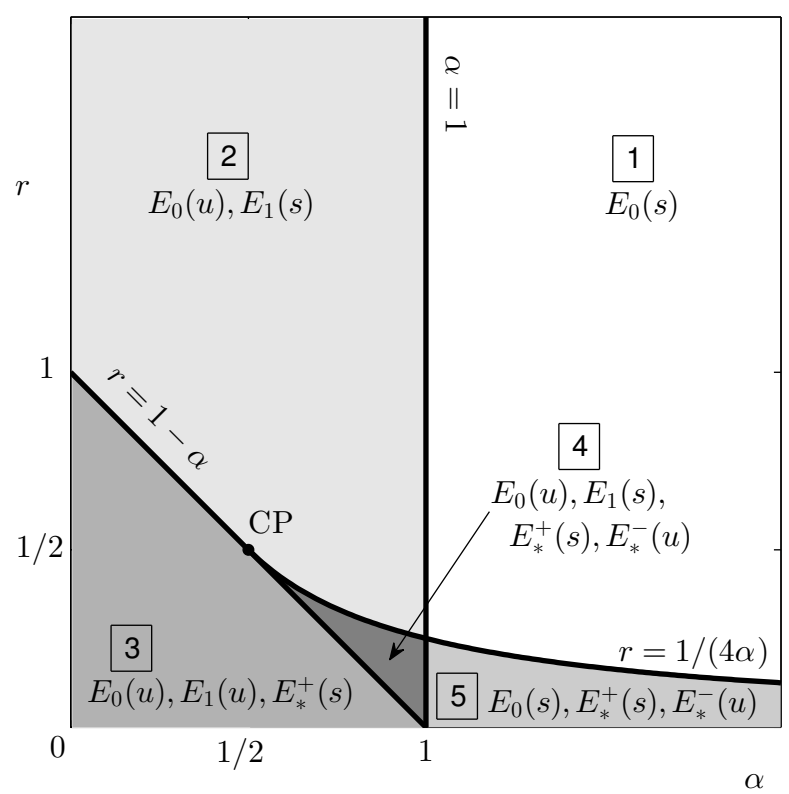

Figure 1: Existence and stability regions of the equilibria given in (6) of the SLM (4) in the parameter plane $(\alpha, r)$. Stable (resp. unstable) equilibria are denoted by "s" (resp. " $u$ "); CP denotes the cusp point.

number of equilibria or for their stability. In three of these regions, labeled by 1,2 and 3 , there exists a single locally stable equilibrium that will be always globally asymptotically stable, in accordance with the theory of cooperative systems [17]. In the two remaining regions 4 and 5 , the bistability occurs. In both regions, the stable manifold of the saddle $E_{*}^{-}$plays the role of separatrix between the basins of attraction of the stable equilibria, while the unstable manifolds provide heteroclinic orbits connecting $E_{*}^{-}$with them. As a consequence, the system can evolve towards a "good" equilibrium $\left(E_{*}^{+}\right)$or a "poor" equilibrium $\left(E_{0}\right.$ or $\left.E_{1}\right)$ equilibrium depending on the initial state. Moreover, when considering interventions in these regions, a parameter change can drive an initial situation either to extinction, or to the absence of high quality green areas only, or to the best coexistence of bio-energy and high quality green areas, depending on the parameter values.

All the boundary curves between these five regions are stationary bifurcation curves. In particular, on the vertical line $\alpha=1$ the system shows transcritical bifurcation points involving the equilibria $E_{1}$ and $E_{0}$. This is also the case of the line $r=1-\alpha$, which involves transcritical bifurcations between the equilibrium $E_{1}$ and a coexistence one $\left(E_{*}^{+}\right.$ or $E_{*}^{-}$, depending on the parameter values), while the curve $r=1 /(4 \alpha)$ is a saddle-node bifurcation curve involving the equilibria $E_{*}^{+}, E_{*}^{-}$. In addition, these last two bifurcation curves intersect at the critical point $C P=(1 / 2,1 / 2)$ in the parameter space, where the three equilibrium states $E_{1}, E_{*}^{+}, E_{*}^{-}$coincide. At this point, the two bifurcation curves share a common tangent; then $C P$ is a mathematical cusp in the $(\alpha, r)$ plane. All these results indicate the presence of a cusp singularity, according to Whitney's theory [18], for the equilibrium surface in the space $(\alpha, r, b)$.

The bifurcation process is described also in Fig. 2, where the values of $b$ at equilibria 


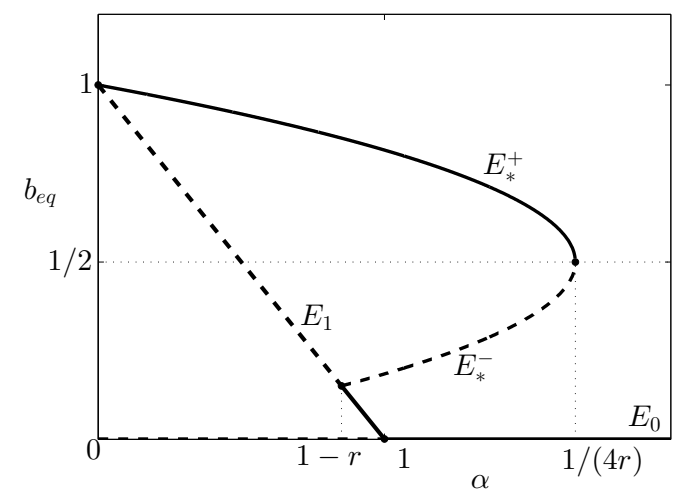

(a) $0<r<1 / 4$

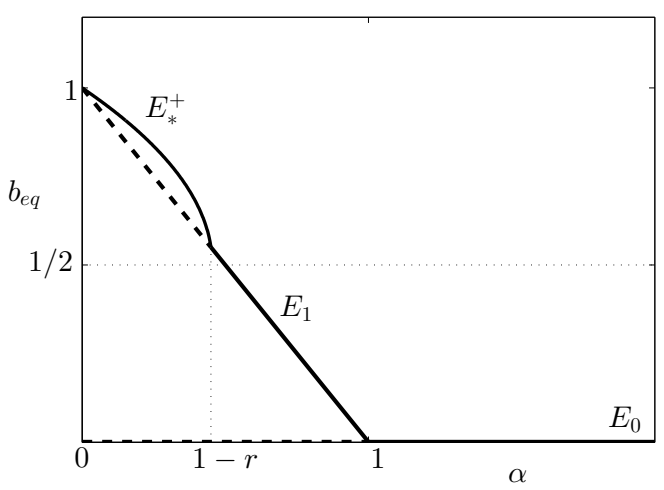

(c) $1 / 2<r<1$

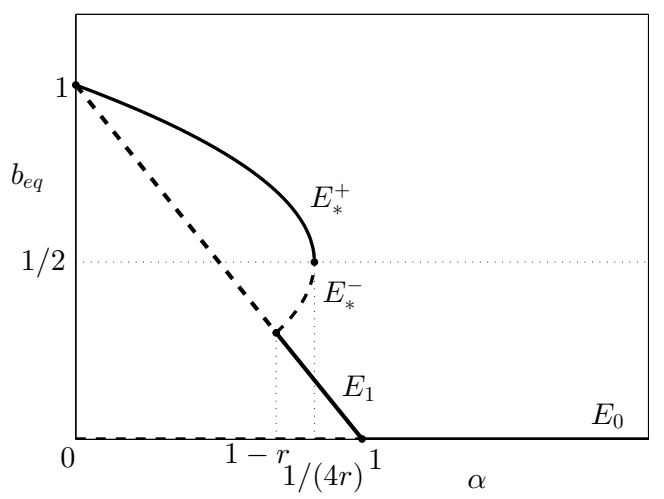

(b) $1 / 4<r<1 / 2$

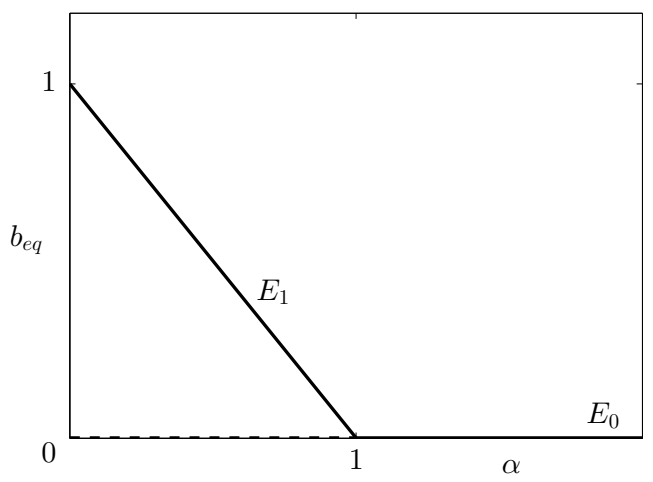

(d) $r>1$

Figure 2: Bifurcation diagrams: equilibrium values of bio-energy $b_{e q}$ versus $\alpha$ for different values of $r$. Solid curves represent stable equilibria, dashed curves represent unstable equilibria. 
are reported versus $\alpha$ for different values of $r$. With reference also to Fig. 1, we can see in detail in Fig. 2 how the equilibrium states collide and disappear. The four panels can be obtained by slicing the diagram in Fig. 1 with lines $r=$ const.

When $r$ is sufficiently small (Fig. 2(a)), by varying $\alpha$ we can pass from region 3 , characterized by the best ecological parameters and where $E_{*}^{+}$is the only attractor, to the region 4 , where the system is bistable with both $E_{*}^{+}$and $E_{1}$ being attractors, then to the region 5 , where bistability is between $E_{*}^{+}$and $E_{0}$, and finally to the region 1 , where $E_{0}$ is the only attractor. By increasing $r$ (Fig. 2(b)) towards $1 / 2$, varying $\alpha$ we pass from regions 3 to 4 by a transcritical bifurcation between $E_{1}$ and $E_{*}^{-}$, then from 4 to 2 by the saddle-node bifurcation involving $E_{*}^{ \pm}$, and finally from regions 2 to 1 by transcritical bifurcation between $E_{1}$ and $E_{0}$. When $r=1 / 2$, the vertex of the parabola, representing the saddle-node bifurcation point, lies on the line $r=1-\alpha$ and we have coincidence of $E_{*}^{ \pm}$and $E_{1}$ (cusp point). For $r$ above $1 / 2$, bistability is no more feasible and the equilibrium $E_{*}^{+}$interacts with $E_{1}$ by transcritical bifurcation as long as $r<1$ (Fig. 2(c)) and by increasing $\alpha$ we cross regions 3,2 and 1 . Finally for $r>1$, there is only a transcritical bifurcation between $E_{1}$ and $E_{0}$ when we pass from region 2 to region 1 .

\section{A preliminary analysis of network dynamics}

In this section we consider the NLM (5) and investigate the effect of the coupling between the LUs. Typically, networks of dynamical systems may present rich behaviors, such as synchronization, periodic solutions, chaos [15]. The NLM (5) is however a system of $2 n$ differential equations of cooperative type [17], since it can be easily proved that its Jacobian matrix has nonnegative off-diagonal entries in the compact invariant hypercube $[0,1]^{2 n}$. Therefore, under mild restrictions [19], it is possible to show that the trajectory of almost every initial state converges to an equilibrium in the compact hypercube and that there are no attracting periodic orbits other than equilibria, because every attractor contains a stable equilibrium.

The analytical study of equilibria for the NLM equations (5) is substantially impracticable; we can only easily verify that the null state is an equilibrium point and using the first Lyapunov criterion, we can investigate its stability. The Jacobian of NLM (5), when evaluated at the null state, is a block diagonal matrix of order $2 n$, with two blocks of order $n$. One block is a diagonal matrix with negative entries $-h_{i} U_{i}$, corresponding to the partial derivatives of the terms on the r.h.s of the equations for the high quality green areas $v_{i}$ with respect to $v_{i}$. The other block is symmetric with the off-diagonal entries equal to $c_{j i}$ and diagonal elements $a_{i}-c_{i}-\ell_{i}$. Thanks to the localization Gershgorin theorem [20], the eigenvalues relevant to this block are bounded from below by $a_{i}-2 c_{i}-\ell_{i}$ and from above by $a_{i}-\ell_{i}=a_{i}\left(1-\alpha_{i}\right)$. Then, if $a_{i}-\ell_{i}<0 \forall i$, namely if the parameter $\alpha$ in each LU is greater than 1 , the null equilibrium state is locally asymptotically stable. This scenario refers to the case in which all LUs belong to regions 1 or 5 (Fig. 1), the only ones where $E_{0}$ is stable for the SLM. Moreover, if there exists an index $i$ such that $c_{i}<\left(a_{i}-\ell_{i}\right) / 2$, then the null equilibrium state is unstable.

To proceed further and get indications about the network asymptotic behavior, we follow a simplified strategy which involves a planar system of ODEs, with the aim of understanding how the connectivity among LUs quantitatively modifies equilibrium states with respect to those of the single LUs. 
In Subsection 4.1 we detail the construction of this simplified model, then in Subsection 4.2 we study its equilibria and their asymptotic stability in terms of two bifurcation parameters, and finally we complete the analysis by characterizing in Subsection 4.3 the stability regions in different parameter planes.

\subsection{The simplified system}

Let $\left(\bar{b}_{i}, \bar{v}_{i}\right), i=1, \ldots, n$ be an equilibrium point for the NLM (5); then, the r.h.s. of NLM equations (5) evaluated at this point is zero, namely

$$
\left\{\begin{array}{l}
a_{i} \bar{b}_{i}\left[1-\bar{b}_{i}\right]-\ell_{i}\left[1-\bar{v}_{i}\right] \bar{b}_{i}-c_{i} \bar{b}_{i}+\bar{I}_{i}=0 \\
d_{i} \bar{v}_{i}\left[1-\bar{v}_{i}\right] \bar{b}_{i}-h_{i} U_{i} \bar{v}_{i}=0 \\
i=1, \ldots n
\end{array}\right.
$$

where the coupling terms have been rewritten in terms of

$$
c_{i}=\sum_{k \in \mathcal{I}_{i}} c_{k i}, \quad \bar{I}_{i}=\sum_{k \in \mathcal{I}_{i}} c_{k i} \bar{b}_{k} .
$$

We remark that the dependence of the $i$-th LU on the neighboring LUs is incorporated in the term $\bar{I}_{i}$, ranging from 0 to $c_{i}$, which is a proper unknown constant at equilibrium for each subsystem relevant to the single LU. In order to get information about the equilibria $\left(\bar{b}_{i}, \bar{v}_{i}\right), i=1, \ldots, n$, and their stability, we consider the simplified assumption that the landscape network is composed by $n-1$ LUs at equilibrium, playing the role of a background, and we connect an additional LU to it (labeled by $i$ ). For a landscape network, it is reasonable to expect that the $n-1$ LUs of the background will not be affected by this insertion. This intuitive assumption deserves however further investigation, that will be matter of future work. Then, the $i$-th LU will relax to equilibrium according to the dynamics given by the system

$$
\left\{\begin{array}{l}
b_{i}^{\prime}(t)=a_{i} b_{i}(t)\left[1-b_{i}(t)\right]-\ell_{i}\left[1-v_{i}(t)\right] b_{i}(t)-c_{i} b_{i}(t)+I_{i} \\
v_{i}^{\prime}(t)=d_{i} v_{i}(t)\left[1-v_{i}(t)\right] b_{i}(t)-h_{i} U_{i} v_{i}(t)
\end{array}\right.
$$

where we have replaced $\bar{I}_{i}$ with an additional generic parameter, let's say $I_{i}$, constant but a priori unknown, dependent on the (unknown) equilibrium values of the bio-energies of the other LUs of the background.

We omit the LU index $i$ in order to simplify the notation, and then consider the simplified system

$$
\left\{\begin{array}{l}
b^{\prime}(t)=a b(t)[1-b(t)]-\ell[1-v(t)] b(t)-c b(t)+I \\
v^{\prime}(t)=d v(t)[1-v(t)] b(t)-h U v(t)
\end{array}\right.
$$

for a generic value of the parameter $I$, with $0 \leq I \leq c$. First we observe that, as for the $\operatorname{SLM}(4), Q=[0,1]^{2}$ is an invariant set for the simplified model and that system (10) has a cooperative structure too, therefore there are no periodic orbits, and the trajectories will converge, eventually monotonically, to an equilibrium. 


\subsection{Equilibria and their asymptotic stability}

Simple calculations lead to equilibrium (the subscript $N$ stands for "network")

$$
E_{1_{N}}=\left(b_{1_{N}}, 0\right)=\left(\frac{a-c-\ell+\sqrt{(a-c-\ell)^{2}+4 a I}}{2 a}, 0\right)
$$

which characterizes agricultural areas, and to two coexistence equilibria

$$
E_{*_{N}}^{ \pm}=\left(b_{*_{N}}^{ \pm}, v_{*_{N}}^{ \pm}\right)=\left(\frac{a-c \pm \sqrt{(a-c)^{2}+4 a(I-a \alpha r)}}{2 a}, 1-\frac{r}{b_{*_{N}}^{ \pm}}\right) .
$$

We notice that the expression of the first component of $E_{1_{N}}$ is found as the positive root of a second order polynomial. The other root is negative for $0<I \leq c$; when $I=0$ the positive root reduces to 0 if $a-c-\ell<0$, otherwise the negative root does it. It follows that, only when $I=0$, the simplified system (10) admits also the null equilibrium state $E_{0 N}=(0,0)$.

As for the single LU model, since $b$ and $v$ are normalized variables, these equilibria are significant only if their components are between 0 and 1 . As regards the equilibrium $E_{1_{N}}$, it is easy to see that it is well defined since the radicand is always positive and also that its first component is positive. In fact, the condition $b_{1_{N}} \leq 1$ is equivalent to $I \leq c+\ell$, which is true by the hypothesis on the parameter $I(I \leq c)$. Therefore, the equilibrium $E_{1_{N}}$ always belongs to $Q=[0,1] \times[0,1]$.

To determine the parameter values for which $E_{*_{N}}^{-}$is admissible we have to impose five conditions: the radicand must be nonnegative and the equilibrium components $b_{*_{N}}^{-}, v_{*_{N}}^{-}$ must range from 0 to 1 . By some calculations, we obtain

$$
E_{*_{N}}^{-} \in Q \Longleftrightarrow\left\{\begin{array} { l } 
{ ( a - c ) ^ { 2 } + 4 a ( I - a \alpha r ) \geq 0 } \\
{ r \leq b _ { * _ { N } } ^ { - } \leq 1 }
\end{array} \Longleftrightarrow \left\{\begin{array}{l}
a(1-r-\alpha)<c \leq a(1-2 r) \\
f(c) \leq I \leq g(c) \\
r<1 / 2
\end{array}\right.\right.
$$

where

$$
f(c)=a\left(\alpha r-\frac{(a-c)^{2}}{4 a^{2}}\right), \quad g(c)=r[a(r+\alpha-1)+c] .
$$

The last condition in (13) is due to the fact that $c$ must be a positive parameter. Moreover, we can find a suitable $c$ only if $a(1-r-\alpha)<a(1-2 r)$, namely $r<\alpha$.

Similarly, the admissibility conditions of the equilibrium $E_{*_{N}}^{+}$are given by

$$
\left\{\begin{array}{l}
(a-c)^{2}+4 a(I-a \alpha r) \geq 0 \\
r \leq b_{*_{N}}^{+} \leq 1
\end{array}\right.
$$

which admits solutions only if $r<1$. After some calculation we obtain

$$
E_{*_{N}}^{+} \in Q \Longleftrightarrow\left\{\begin{array} { l } 
{ c \geq a ( 1 - 2 r ) } \\
{ I \geq g ( c ) } \\
{ r < 1 }
\end{array} \quad \vee \left\{\begin{array}{l}
c<a(1-2 r) \\
I \geq f(c) \\
r<1 / 2
\end{array}\right.\right.
$$


Remark 2. It can be noticed that a necessary condition for the admissibility of both $E_{*_{N}}^{+}$and $E_{*_{N}}^{-}$is $r<1$, namely $h U<d$, which means that both intensity and dispersion of built-up areas are sufficiently low. This fact is in accordance with the comments in Remark 1 for the single LU model. As in the single case, standard calculations show that the bio-energy value of the coexistence equilibrium $E_{*_{N}}^{+}$is always greater than the bio-energy value of $E_{1_{N}}$, namely $b_{*_{N}}^{+}>b_{1_{N}}$.

\section{Proposition 2.}

(i) $E_{0 N}$, admissible for $I=0$, is locally asymptotically stable if and only if $a-c-\ell<0$.

(ii) $E_{1_{N}}$ is locally asymptotically stable if and only if $\left\{\begin{array}{l}c>a(1-r-\alpha) \\ 0<I<g(c) .\end{array}\right.$

(ii) $E_{*_{N}}^{+}$is locally asymptotically stable, while $E_{*_{N}}^{-}$is a saddle (when they are admissible).

Proof. In order to study the local stability we evaluate the Jacobian matrix of the simplified system (10)

$$
J=\left(\begin{array}{cc}
a[(1-2 b)-\alpha(1-v)]-c & \ell b \\
d v(1-v) & d[b(1-2 v)-r]
\end{array}\right)
$$

at each equilibrium and determine the sign of its eigenvalues.

(i) The Jacobian matrix evaluated at $E_{0 N}$ is a diagonal matrix with eigenvalues $\lambda_{1}=$ $a-c-\ell$ and $\lambda_{2}=-d r$.

(ii) The Jacobian matrix evaluated at $E_{1_{N}}$ is an upper triangular matrix, whose eigenvalues are $\lambda_{1}=a\left(1-\alpha-2 b_{1_{N}}\right)-c$ and $\lambda_{2}=d\left(b_{1_{N}}-r\right)$. By simple calculations we find that the first eigenvalue is always negative, while the second one is negative if and only if $b_{1_{N}}<r$. Such condition, by some algebra, expresses the thesis.

(iii) The Jacobian matrix evaluated at the coexistence equilibria can be written in the compact form

$$
J\left(E_{*_{N}}^{ \pm}\right)=\left(\begin{array}{cc}
a\left(1-\alpha r / b_{*_{N}}^{ \pm}-2 b_{*_{N}}^{ \pm}\right)-c & \ell b_{*_{N}}^{ \pm} \\
d v_{*_{N}}^{ \pm}\left(1-v_{*_{N}}^{ \pm}\right) & d\left(r-b_{*_{N}}^{ \pm}\right)
\end{array}\right) .
$$

The determinant, by simple calculations, results to be:

$$
\operatorname{det}\left(J\left(E_{*_{N}}^{ \pm}\right)\right)=d\left(a-c-2 a b_{*_{N}}^{ \pm}\right)\left(r-b_{*_{N}}^{ \pm}\right) .
$$

The last term of this product is always negative, due to the admissibility conditions (13) and (15). Moreover, after some calculations, the second term of the product, $\left(a-c-2 a b_{*_{N}}^{ \pm}\right)$, can be reduced to $\mp \sqrt{(a-c)^{2}+4 a(I-a \alpha r)}$. Therefore the sign of the determinant is constant, in particular $J\left(E_{*_{N}}^{-}\right)$is negative (and therefore $E_{*_{N}}^{-}$is a saddle), while $J\left(E_{*_{N}}^{+}\right)$ is positive. To determine the stability of $E_{*_{N}}^{+}$we have to study the sign of the trace of $J\left(E_{*_{N}}^{+}\right)$, which can be written as:

$$
\begin{aligned}
\operatorname{tr}\left(J\left(E_{*_{N}}^{+}\right)\right) & =a\left(1-\alpha r / b_{*_{N}}^{ \pm}-2 b_{*_{N}}^{ \pm}\right)-c+d\left(r-b_{*_{N}}^{ \pm}\right) \\
& =-a \alpha r / b_{*_{N}}^{ \pm}+d\left(r-b_{*_{N}}^{ \pm}\right)-\sqrt{(a-c)^{2}+4 a(I-a \alpha r)}
\end{aligned}
$$

which is negative, since all its addends are negative, and then the thesis holds. 
Remark 3. The case $I=0$ for the simplified system (10) is strictly related to the null equilibrium of the whole network, discussed at the beginning of this Section. In fact, $I=0$ can be obtained only if the background is at the null state and it is remarkable that the stability condition for $E_{0 N}$ is analogous to the ones found for the stability of the null equilibrium state for the network.

\subsection{Stability regions}

The stability analysis of the system (10) turns out to depend also on the additional parameters $c$ and $I$, besides $r$ and $\alpha$. The representation of the behaviors of the system in terms of equilibria and their stability is then much more complicated and it is schematized in Fig. 3. In the parameters plane $(\alpha, r)$ (panel (a)) we identify six different regions, which present different scenarios. Regions $A-E$ are represented in panels (b)-(f) in the parameters plane $(c, I)$, respectively, while region $F$ is not further detailed since only the equilibrium $E_{1_{N}}$ is admissible and it is locally asymptotically stable (and also globally stable, according to the theory of cooperative systems which holds for system (10)). In each panel (b)-(f) different tonalities represent different number of admissible equilibria or different stability properties. In particular, white regions denote sets of non admissible parameter values, since admissibility requires $I \leq c$; in the light gray regions there exists only the equilibrium $E_{1_{N}}$ and it is locally (and globally) asymptotically stable; in the gray regions there exist the equilibria $E_{1_{N}}$ (unstable) and $E_{*_{N}}^{+}$(asymptotically stable); in the dark gray regions there exist the equilibrium $E_{1_{N}}$ (locally asymptotically stable) and both coexistence equilibria $E_{*_{N}}^{+}$(locally asymptotically stable) and $E_{*_{N}}^{-}$(unstable).

Remark 4. If we analyze the effect of the coupling on the position of the equilibria, we find that if the constant input $I$ is sufficiently high, then the bio-energy at equilibrium of the simplified model (10) is greater than the corresponding value of the single LU, in fact

$$
b_{1_{N}}>b_{1} \quad \Longleftrightarrow \quad I>c(1-\alpha)=c b_{1}
$$

This is also the case of the equilibrium $E_{*_{N}}^{+}$, whenever it is admissible, in fact by some algebra we get

$$
b_{*_{N}}^{+}>b_{*}^{+}, \quad v_{*_{N}}^{+}>v_{*}^{+} \Longleftrightarrow I>c b_{*}^{+} .
$$

On the contrary, the bio-energy and the fraction of high quality areas of the equilibrium $E_{*_{N}}^{-}$, whenever it is admissible, are greater than their respective values of the isolated LU if $I$ is sufficiently small, namely

$$
b_{*_{N}}^{-}>b_{*}^{-}, \quad v_{*_{N}}^{-}>v_{*}^{-} \quad \Longleftrightarrow \quad I<\min \left\{c b_{*_{N}}^{-}, \frac{a c-c^{2}}{2 a}\right\} .
$$

The coupling can also force the system to tend to a different equilibrium type. It can be seen that

$$
b_{1_{N}}>b_{*}^{+} \Longleftrightarrow I>b_{*}^{+}(c+\ell)-\ell r,
$$

namely that the bio-energy $b_{1_{N}}$ of the simplified model is greater than the bio-energy $b_{*}^{+}$ of the single LU if $I$ is sufficiently high.

A first consequence of this analysis is that the sectors that belong to region $F$ in the $(\alpha, r)$ plane (see Fig. 3(a)) can evolve only towards the equilibrium $E_{1_{N}}$ regardless of the values of $c$ and $I$, while in the single $L U$ model they can reach either the equilibrium $E_{0}$ or the equilibrium $E_{1}$ (see Fig. 1), depending on the initial data. 
As it can be seen in Figs. 3(b)-3(f), in all the other regions $A-E$ the number and the stability of equilibria do not depend only on $\alpha$ and $r$. Nevertheless, for regions $A$, $B$, $D$ and $E$ we can still find conditions on the parameter $c$ for which the system admits only one stable equilibrium, regardless of $I$. More specifically, it is possible to find a threshold $\tilde{c}$, that depends on the region, below which either $E_{1_{N}}$ or $E_{*_{N}}^{+}$are locally asymptotically stable. Therefore in this case, given the set of parameters and since $c$ is a geometric parameter that can be calculated from the connectivity indices with formula (9), we can a priori determine to which equilibrium the system will tend, without knowing I. As an example, let us consider region $A$ of Fig. 3(a). For these values of $\alpha$ and $r$ the isolated system only admits a stable equilibrium, namely $E_{*}^{+}$(see Fig. 1). For the simplified model (10), as it can be seen by Fig. 3(b) and due to the previous considerations, if $c<\tilde{c}=a(1-r-\alpha)$ then the trajectory tends to the coexistence equilibrium $E_{*_{N}}^{+}$, of the same type of the isolated case. On the contrary, if $c>\tilde{c}$, either the equilibrium $E_{1_{N}}$ or $E_{*_{N}}^{+}$could be locally asymptotically stable, depending on the value of $I$. Similar comments hold for the other regions, except for region $C$, where for each $c$ both equilibria $E_{1_{N}}$ and $E_{*_{N}}^{+}$can be stable, depending on $I$. Moreover, as can be seen in Figs. 3(c), 3(d), 3(e), there are bistability regions (dark gray color) in which the estimated $I$ does not determine univocally the attractor of the system.

\section{Application to a case study in Northern Italy}

We consider an environmental system located in the northern side of the Turin Province (Italy). We focus in particular on five LUs of this region where rather compact built-up territorial patches interact with natural reserve areas. Table 1 provides the initial data and the values of the parameters (indicators) that identify the different ecological sectors of the territory under investigation; Table 2 contains the geometric parameters that characterize the borders of the LUs. The data have been obtained from the GIS measurements in the master degree thesis [16], where a territory of 24 LUs centered around the municipality of Cirié (Turin) has been taken into account. For the purposes of the present paper, which is mainly devoted to show the effects of connectivity between different patches of a territory, we have selected only 5 sectors which better fit our aims since the remaining other 19 sectors, not here examined, are generally not well connected to the five LUs we have chosen. The data of Table 1 can be commented in order to better characterize each $\mathrm{LU}$, in the sense that the numerical values of the indicators highlight immediately some peculiarities of each LU. In fact, LU18 and LU20 present a good production of BTC $\left(b_{0}\right)$ together with a rather high value of solar exposure $(a)$. Conversely, LU19 has a low value of high ecological quality green area $\left(v_{0}\right)$ and a strong intensity of construction $(U)$ which of course implies high presence of barriers (high value of $\ell$ ). A peculiarity characterizing LU20 is a low value of construction which conversely is strongly dispersed $(h=2.2)$. By examining also Table 2 it comes out the peculiarity of the connection between LU17 and LU19 which present a connectivity index equal to zero: this is not surprising, since these two sectors are completely divided by two contiguous cities.

On the basis of these data we can know a "priori" how many stable equilibria are present in each (isolated) LU, as represented in Fig. 4(a) by the points which locate the sectors in the proper region of the $(\alpha, r)$ plane. The positions of sectors LU18 and LU1 in the $(\alpha, r)$ plane are quite close to each other, and their dynamical behaviors are expected to be very similar. 


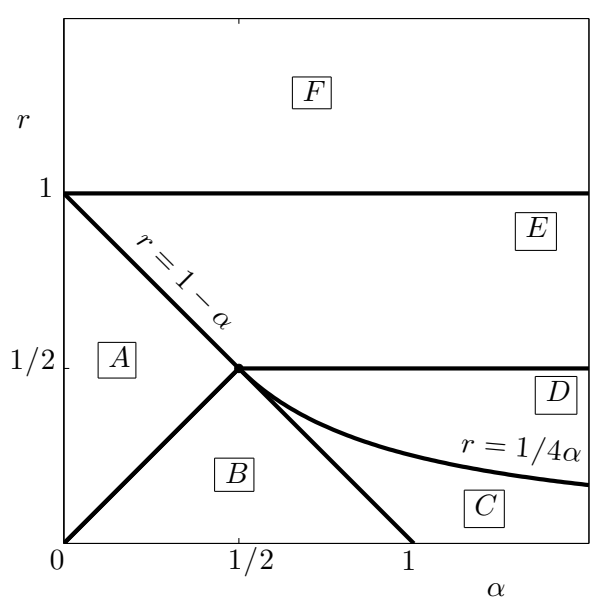

(a) $(\alpha, r)$ plane

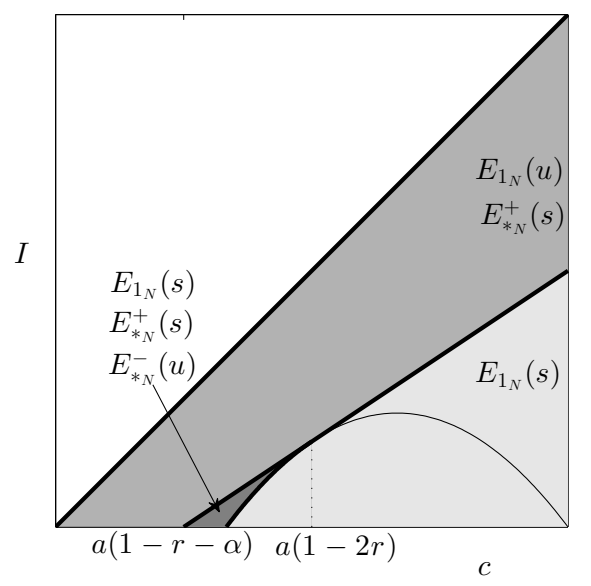

(c) Region $B-(c, I)$ plane

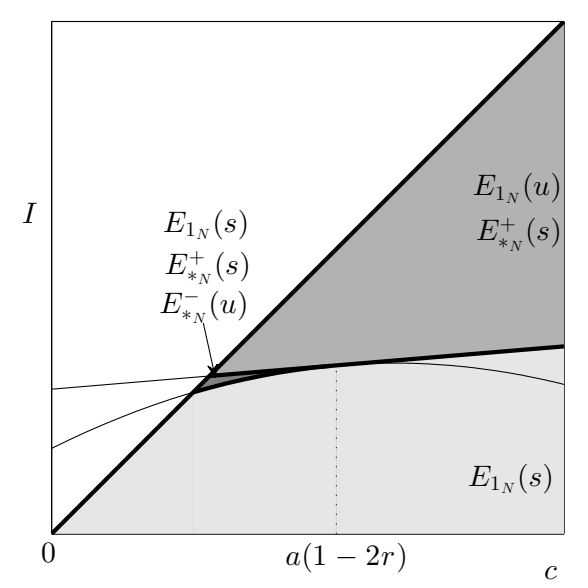

(e) Region $D-(c, I)$ plane

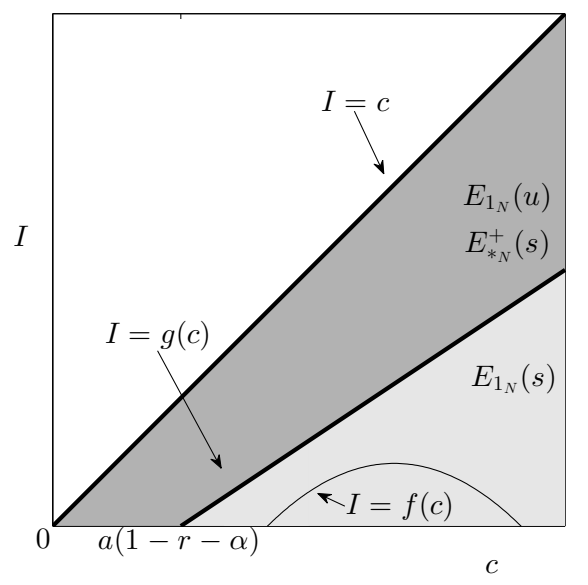

(b) Region $A-(c, I)$ plane

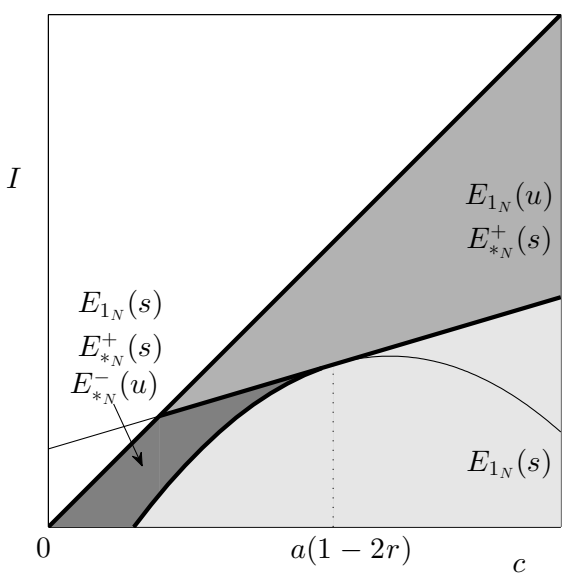

(d) Region $C-(c, I)$ plane

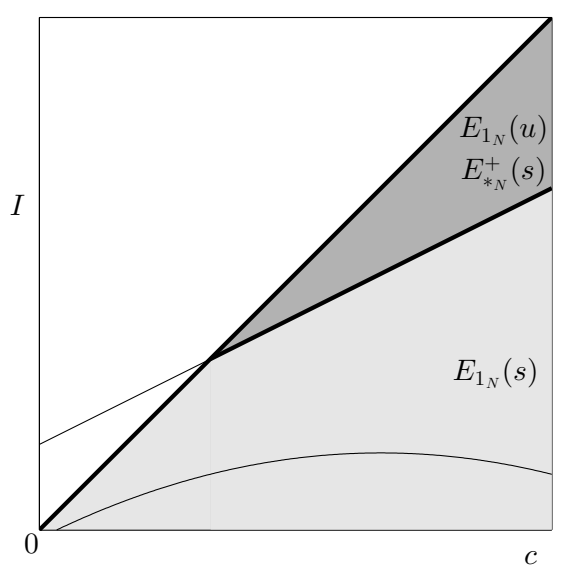

(f) Region $E-(c, I)$ plane

Figure 3: Existence and stability regions of the equilibria given in (11)-(12) for the simplified model (10). Curves $I=f(c)$ and $I=g(c)$ are defined in (14). Light gray regions: $E_{1_{N}}$ locally asymptotically stable. Gray regions: $E_{1_{N}}$ (unstable) and $E_{*_{N}}^{+}$(locally asymptotically stable). Dark gray regions: $E_{1_{N}}$ (locally asymptotically stable), $E_{*_{N}}^{+}$(locally asymptotically stable) and $E_{*_{N}}^{-}$(unstable). 


\begin{tabular}{|c||r|c|c|c|c|c|c|c|c|c|c|}
\hline $\mathbf{L U}$ & \multicolumn{1}{|c|}{$S_{i}$} & $P_{i}$ & $b_{i 0}$ & $v_{i 0}$ & $a_{i}$ & $\ell_{i}$ & $d_{i}$ & $h_{i}$ & $U_{i}$ & $\alpha_{i}=\ell_{i} / a_{i}$ & $r_{i}=h_{i} U_{i} / d_{i}$ \\
\hline \hline $\mathbf{1}$ & 19156648 & 25248 & 0.19 & 0.30 & 0.39 & 0.05 & 0.35 & 1.83 & 0.09 & 0.128 & 0.471 \\
\hline $\mathbf{1 7}$ & 6369795 & 12599 & 0.13 & 0.26 & 0.32 & 0.11 & 0.26 & 1.58 & 0.21 & 0.344 & 1.276 \\
\hline $\mathbf{1 8}$ & 69754645 & 60482 & 0.34 & 0.31 & 0.44 & 0.04 & 0.40 & 1.97 & 0.07 & 0.091 & 0.345 \\
\hline $\mathbf{1 9}$ & 4589299 & 18604 & 0.17 & 0.13 & 0.31 & 0.32 & 0.28 & 1.88 & 0.61 & 1.032 & 4.096 \\
\hline $\mathbf{2 0}$ & 42953048 & 38826 & 0.45 & 0.30 & 0.51 & 0.05 & 0.26 & 2.20 & 0.09 & 0.098 & 0.761 \\
\hline \hline
\end{tabular}

Table 1: Data of the landscapes in the considered environmental system.

\begin{tabular}{|c||r|c|c|}
\hline Interacting LUs & $H_{k i}$ & $L_{k i}$ & $c_{k i}=H_{k i} / L_{k i}$ \\
\hline \hline $\mathbf{1}-\mathbf{1 7}$ & 3017 & 7543 & 0.4 \\
\hline $\mathbf{1}-\mathbf{1 8}$ & 3664 & 9543 & 0.38 \\
\hline $\mathbf{1 7}-\mathbf{1 8}$ & 5429 & 8671 & 0.63 \\
\hline $\mathbf{1 7}-\mathbf{1 9}$ & 0 & 5421 & 0 \\
\hline $\mathbf{1 8}-\mathbf{1 9}$ & 3352 & 8953 & 0.37 \\
\hline $\mathbf{1 8}-\mathbf{2 0}$ & 5390 & 10780 & 0.5 \\
\hline $\mathbf{1 9}-\mathbf{2 0}$ & 1553 & 3106 & 0.5 \\
\hline
\end{tabular}

Table 2: Geometric parameters and connectivity indices that describe the interaction between the LUs.

The network composed by the five LUs, modeled by the NLM equations (5), is characterized by the adjacency matrix

$$
C=\left[\begin{array}{ccccc}
0 & c_{17,1} & c_{18,1} & 0 & 0 \\
c_{1,17} & 0 & c_{18,17} & c_{19,17} & 0 \\
c_{1,18} & c_{17,18} & 0 & c_{19,18} & c_{20,18} \\
0 & c_{17,19} & c_{18,19} & 0 & c_{20,19} \\
0 & 0 & c_{18,20} & c_{19,20} & 0
\end{array}\right]
$$

where $c_{k i}=c_{i k}$ are the connectivity indices that describe how the LUs interact to each other; their numerical values, for the network under consideration, follow from the geometric parameters reported in Table 2.

In Figs. 4 we present the trend to equilibrium of each LU when the initial data are the ones reported in Table 1. More precisely, we compare the time evolution of the state variables $(b, v)$ of each LU under investigation when it is connected (black solid lines, solutions to the NLM equations (5)) or isolated (gray dashed lines, solutions to SLM equations (4)), with parameters and initial conditions given in Tables 1 and 2. These figures show the effect of the coupling in the network model and its ability to modify or not the asymptotic behaviors of the single LU. As we can see, there are situations in which the network drives a sector towards a better scenario, characterized by higher level of green areas and bio-energy, but this is not the rule. More precisely, LU17, when connected to the others, evolves towards the same qualitative (single) scenario of predominant agricultural production, with however a slight improvement in the level of bio-energy; both LU1 and 
LU18 as a part of the network evolves towards the same kind of scenario, that is good quality of bio-energy in presence of a certain amount of high quality areas, but the values of the components of the network equilibrium point relevant to LU1 and LU18 are both worse than the corresponding values in the single LU model. In sectors LU19 and LU20 there is a more evident difference in the asymptotic behaviors, since the network causes a change of the attractor: in the single model, LU19 tends to gradually loose its ecological quality in presence of a strong landscape fragmentation, while it evolves towards the agricultural scenario when connected to the other sectors, improving its quality; on the contrary, LU20 gets worse in the network, evolving towards the agricultural scenario when instead would reach the high quality equilibrium in absence of the network. We point out that in this case study the null state is not reached by the network, even if it could be locally stable; in other words, such environmental system takes advantage of the coupling. As a further remark, we can see in Fig. 4(e) for LU19 that the time evolution of the state variable $v$ in the single LU model is almost indistinguishable from that in the network model. A possible explanation is that since $r_{19}$ is rather large (see Table 1 ), the differential equation for the variable $v_{19}$ in both SLM (4) and NLM (5) has a dominant term that does not contain the variable $b_{19}$ (that is the variable influenced by the connected LUs):

$$
v_{19}^{\prime}(t)=d_{19}\left[v_{19}(t)\left(1-v_{19}(t)\right) b_{19}(t)-r_{19} v_{19}(t)\right] \approx-d_{19} r_{19} v_{19}(t)
$$

and then the same exponential decrease can be expected for the single LU model and for the network system. This comment also applies to the $v$ variable relevant to LU17 (see Fig. 4(c)). Summing up, the interactions within the network improve the dynamics of LUs 17 and 19 and, at the same time, deteriorate the dynamics of LUs 1, 18 and 20.

In Fig. 5 we compare the trajectories in the phase subspace $(b, v)$ obtained by integrating the SLM equations (4) (dashed gray lines), the NLM equations (5) (black solid lines) and also that of the simplified model (10) (gray solid lines). The value of $I$ (one for each LU) used in the simplified model has been numerically evaluated a posteriori, according to (9), from the NLM simulation when the network has reached its equilibrium (with parameters and initial data given in Tables 1 and 2). It is remarkable that the simplified and the network models evolve towards the same equilibrium states in each LU, as expected; relaxation to equilibrium for the simplified model is instead different with respect to the one prescribed by the NML equations, since the simplified model describes essentially the dynamics of each LU when inserted in a network at equilibrium (background), thus under particular assumptions on the relaxation times. The relation between the trend-to-equilibrium dynamics of the simplified and NLM systems deserves however further investigation, that will be matter of future work.

As an additional test of validity of our simplification, we have numerically checked the expression (11) and (12) of the equilibrium states for the parameter values of Tables 1 and 2. To do this we have integrated the NLM equations (5) and, as already discussed, each LU reaches a steady state (Fig. 5). Then we have computed the value of $I$ in system (10) of the simplified model at the network equilibrium, and we have obtained a value for the equilibria (11) and (12) for each LU. The obtained values turn out to be in agreement with the equilibrium values obtained integrating the NLM equations (5), with an error of order $10^{-8}$ due to numerical approximations.

The behavior of the NLM can be explained on the basis of the analysis performed in Section 4 for the simplified system (10). In Figure 6(a) the position of each LU in the $(\alpha, r)$ plane for our case study is shown. In particular, sectors LU17 and LU19 belong to 
region $F$ in the $(\alpha, r)$ plane. This fact explains a priori the change of equilibrium type to which LU19 converges: the trajectory tends to $E_{0}$ in the single LU model and to $E_{1_{N}}$ in the coupled system, as can be seen in Fig. 5(d). Also sector LU17 belongs to region $F$ and it tends to the equilibrium denoting an agricultural scenario both in the single LU model and in the network case. Moreover, from the simulations we observe that the value $b_{1_{N}}$ of the bio-energy at the equilibrium for LU17 in the coupled case is greater than $b_{1}$ of the isolated one. This is in agreement with the condition (16) where $I=I_{17}$ is estimated a posteriori using the simulation outcomes and formula (9), and turns out to be greater than $c b_{1}$.

The other sectors, instead, belong to region $A$ and, as it can be seen in Fig. 6(b), the connectivity values $c_{1}, c_{18}$ and $c_{20}$ (dashed lines) are greater than the respective thresholds $\tilde{c}$, so we have to compute $I_{1}, I_{18}$ and $I_{20}$ using the simulation outcomes and formula (9) to determine the stable equilibrium. We find that parameters of sectors LU1 and LU18 lie in the gray region, so they evolve to the coexistence equilibrium $E_{*_{N}}^{+}$, while the parameters of LU20 are in the light gray region, therefore the trajectories tend to $E_{1_{N}}$. This is in agreement with the network simulations reported in Fig. 5 and more specifically it explains the change of equilibrium type of LU20.

Thanks to the theoretical study of the simplified model (10) and in particular to the Remark 4, we can justify the decrease in the bio-energy at the equilibrium from the single LU model to the network one, observed for sectors LU1, LU18 and LU20. In fact, the computed values of $I$ in such cases are smaller than the respective thresholds in conditions (17) and (18).

\section{Concluding remarks}

In the present paper we have proposed a model for an environmental system, distributed in several sectors (LUs) as an ecological network, using as state variables the production of bio-energy and the percentage of high ecological quality areas in each sector. The LUs are coupled to each other by a linear interaction term proportional to the difference between the bio-energies and constitute a network of dynamical systems. First we have considered the single LU model and analyzed its evolution towards a stable equilibrium state. Then we have focused our attention on the network model. Our analysis has shown that the interaction between the LUs can lead each ecological sector both to a better or to a worse ecological scenario, with respect to the single LU model, depending on the values of the state variables of the nearest LUs. In fact, the case study has shown clearly that LU1 and LU18 commute to a worse situation when they are connected in the network. On the contrary, LU17 improves its bio-energy value. In addition, LU19 and LU20 present even a change of equilibrium state passing from an attractor to another one: these behaviors can be interpreted as examples of different kinds of resilience of landscape systems under the effect of environmental stress.

The simplified system, representing the situation of a single LU added to a network at the equilibrium (background), has proved to be an useful tool of investigation of the network. For instance, the simplified model univocally determines the asymptotic behavior of sectors LU17 and LU19, regardless of the initial conditions.

In our opinion future perspectives and developments may involve the modification of the connectivity term in order to better detail the interaction. In particular, we plan to consider time-dependent connectivity parameters $c_{i j}(t)$ that depend on the bio-energy 


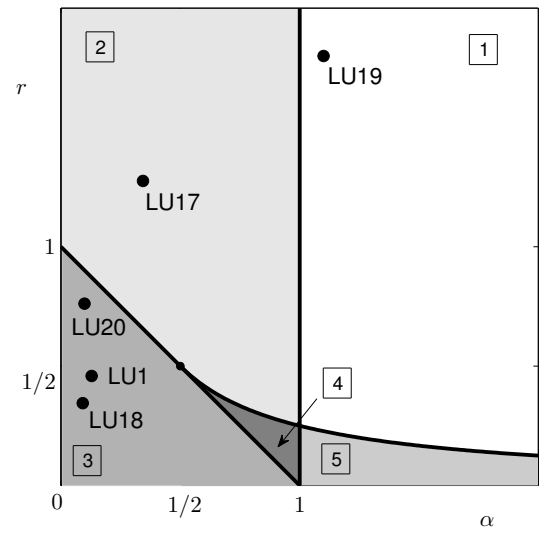

(a)
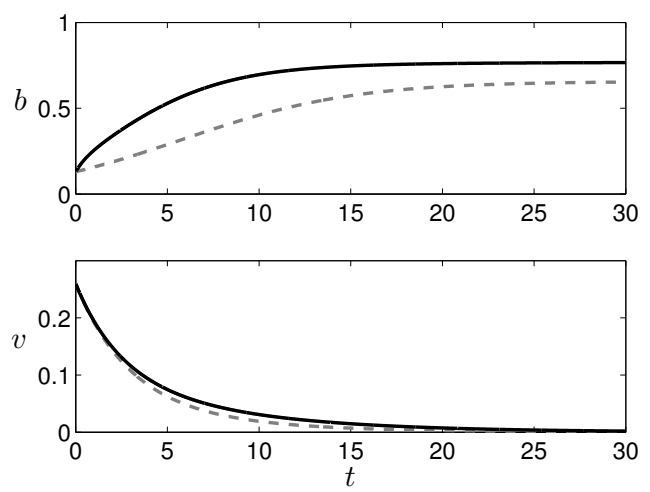

(c) LU17
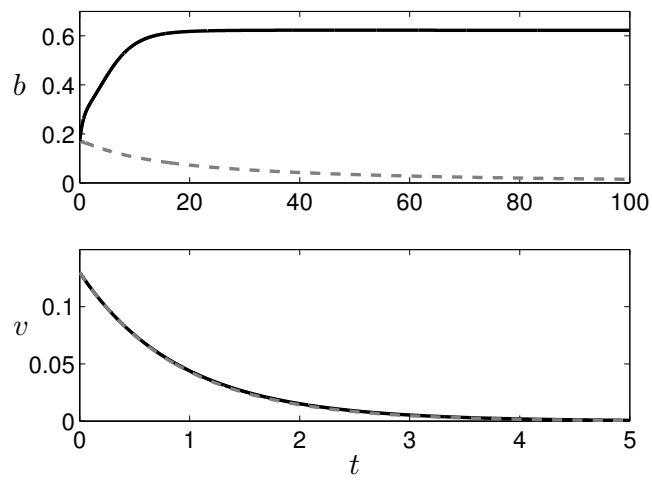

(e) LU19
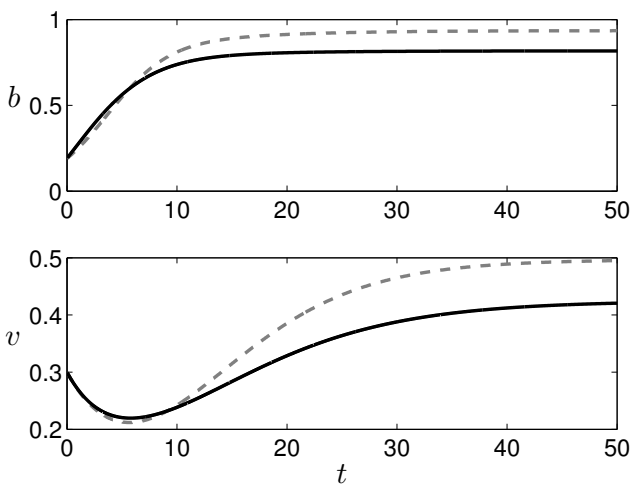

(b) LU1
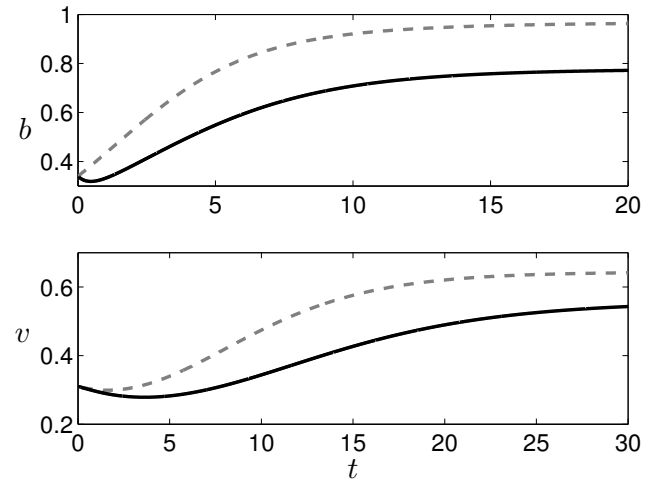

(d) LU18
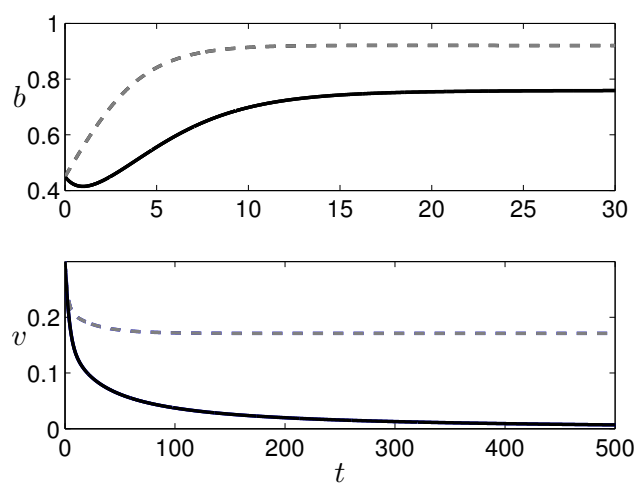

(f) LU20

Figure 4: Panel (a): Black points indicate the position in the $(\alpha, r)$ plane of the five considered LUs belonging to the northern Turin Province, whose parameters are reported in Table 1. The LUs belong to regions $1,2,3$ in which there is only one stable equilibrium of the single LU model, namely $E_{0}, E_{1}, E_{*}^{+}$ respectively. Panels (b)-(f): time evolution of the solutions $b(t)$ and $v(t)$ of the SLM equations (4) (dashed lines) and for the NLM equations (5) (solid lines) for the LUs 1, 17, 18, 19 and 20. LU1, LU17, LU18 evolve towards the same kind of scenario in the two cases; LU19, LU20 reach different scenarios when connected in the network. 


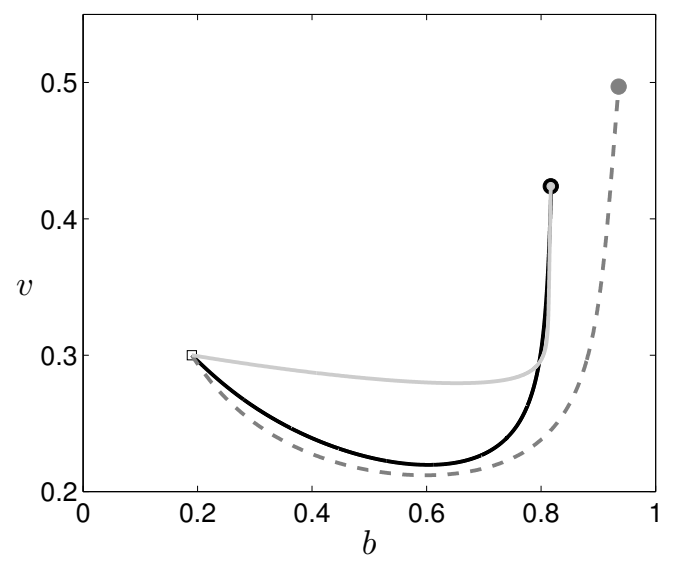

(a) LU1

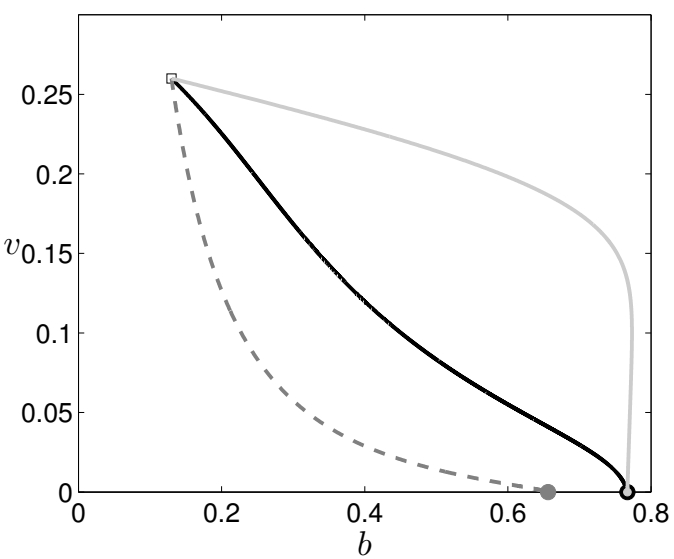

(b) LU17

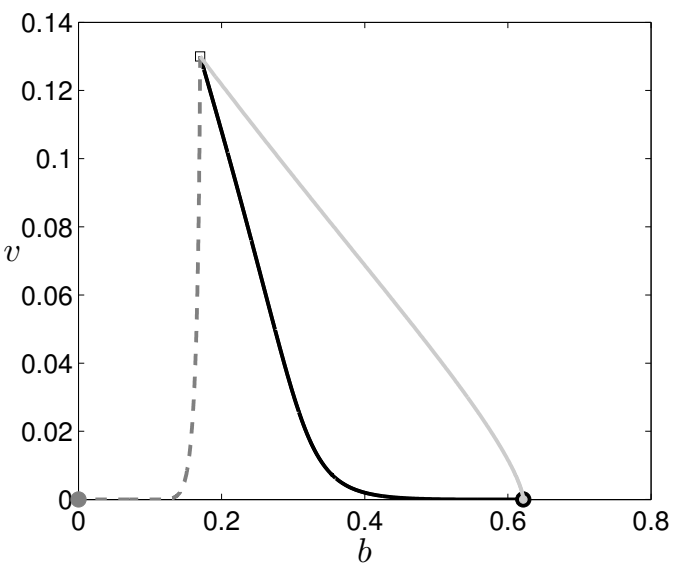

(d) LU19

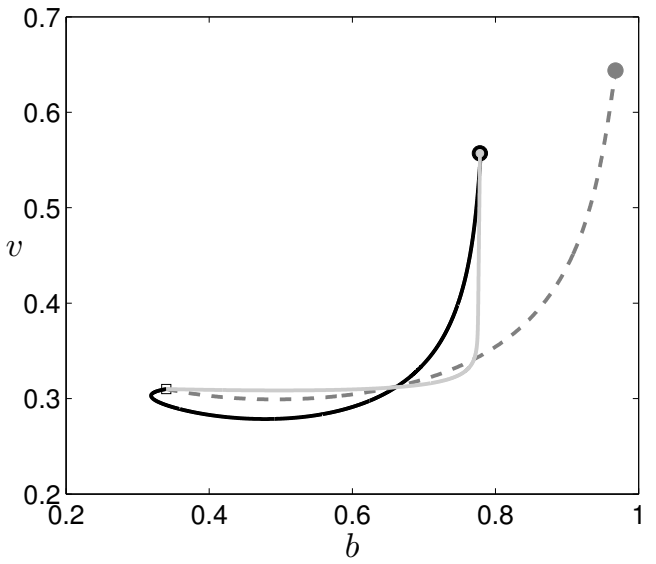

(c) LU18

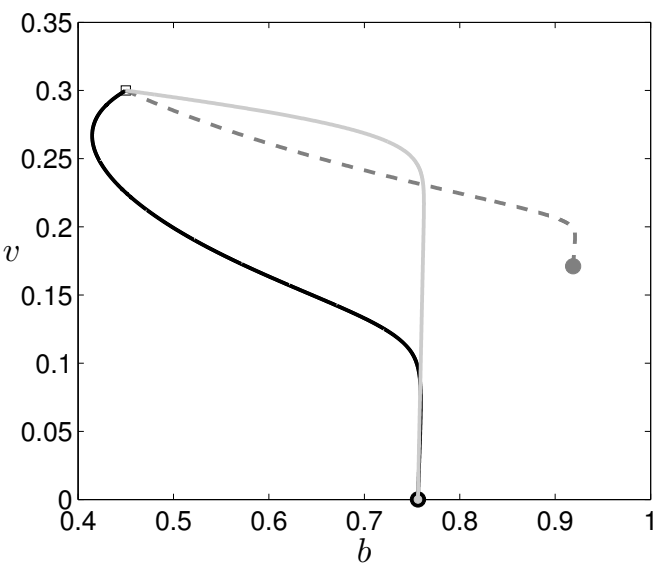

(e) LU20

Figure 5: Trajectories in the phase subspace of each LU for the SLM (4) (dashed gray lines), for the NLM (5) (solid black lines) and for the simplified model (10) (solid gray lines); squares indicate initial conditions; points denote the steady states. 


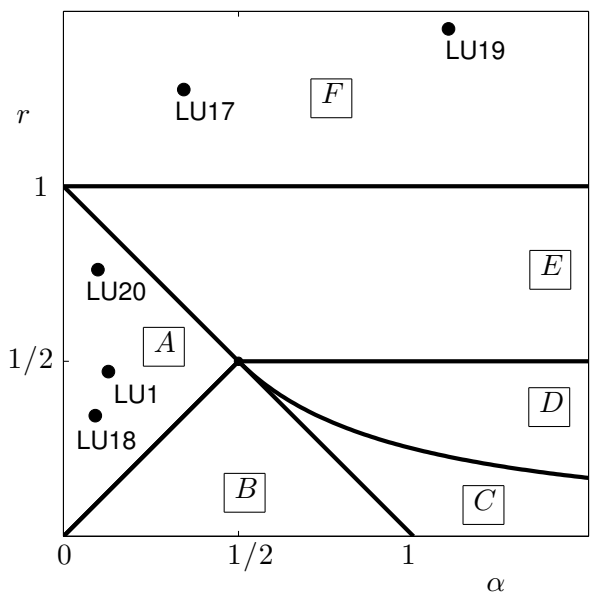

(a) $(\alpha, r)$ plane

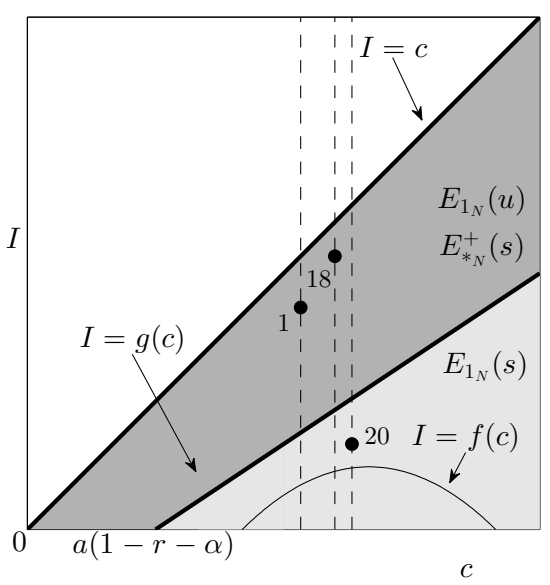

(b) $(c, I)$ plane

Figure 6: Panel (a): Black points indicate the position in the $(\alpha, r)$ plane of the five considered LUs belonging to the northern Turin Province, whose parameters are reported in Table 1. Panel (b): Position in the $(c, I)$ plane of the three LUs belonging to region $A$ of the $(\alpha, r)$ plane. This is a qualitative figure, since the position of the curves $I=f(c)$ and $I=g(c)$ depends on the chosen LU.

flux through the boundaries, similarly to what has been proposed in paper [14] where the connectivity index is defined in a different way.

Furthermore, another modification to the model could take into account a coupling term also in the differential equations relevant to the $v_{i}$ variables. More specifically, a loss term on the $i$-th LU depending on the extension of the low ecological quality areas of its neighbors (namely proportional to $1-v_{k}(t)$, for $k \in \mathcal{I}_{i}$ ) could be considered. This term should describe the negative impact of the low quality areas of the LUs surrounding the $i$-th itself. Conversely, also a gain term, accounting for an increase in the quality of the ecological sector, can be introduced, in order to take into account the pollination phenomena that eventually may arise thanks to the presence of high quality areas in the nearby LUs.

We expect that these improvements of the model lead to a more detailed and realistic description of the phenomena characterizing an environmental system, even if the analysis developed in this paper shows that a simple linear coupling term is able to reproduce some relevant aspects of such an interaction. Therefore, these new models can be employed to aid the management and the decision process in defining planning strategies by the prediction of possible future scenarios of the environment and to help in preventing incidental risks of the territory.

\section{Acknowledgments}

The authors are grateful to the anonymous referees for their valuable comments and suggestions which helped them to considerably improve the paper. The present research has been partially supported by Portuguese Funds FCT Project UID/MAT/00013/2013, and by the Italian National Group GNFM of INdAM. One of the Authors (AJS) thanks the Italian institution for the financial support given in her visiting professor program in Italy. 


\section{Appendix A}

We report here the definitions with reference to paper [13] of the parameters $a_{i}$ and $d_{i}$ appearing in the Eqs. (2)-(3). We have

$$
a_{i}:=\frac{w_{1} S_{i}^{S E S}+w_{2} S_{i}^{W}+w_{3} S_{i}^{N E}}{S_{i}} \leq 1
$$

where the $w$ s are suitable weights and $S_{i}^{S E S}, S_{i}^{W}, S_{i}^{N E}$ are the soil surfaces with exposure to south-east-south, west and north-east, respectively. Moreover

$$
d_{i}=\frac{1}{3}\left(a_{i}+k_{i}^{h u}+k_{i}^{e c}\right) \leq 1, \quad k_{i}^{h u}=\frac{w_{4} S_{i}^{h}+w_{5} S_{i}^{s}}{S_{i}}, \quad k_{i}^{e c}=1-P_{i} / \sum_{j=1}^{m_{i}} P_{j i},
$$

where $k_{i}^{h u}$ and $k_{i}^{e c}$ stand for the indices of relative humidity and ecotonal length, $S_{i}^{h}$ and $S_{i}^{s}$ being, respectively, the areas of soil characterized by humidity and sub-humidity; moreover $P_{j i}$ is the length of the $j$-th biotope perimeter, and $P_{i}$ that of the $i$-th LU.

\section{References}

[1] Lotka AJ. Contribution to the energetics of evolution. Proceedings of the National Academy of Sciences of the United States of America 1922; 8(6): 147-151.

[2] Ingegnoli V. Landscape Ecology: a Widening Foundation. Berlin: Springer-Verlag; 2002.

[3] Li BL. Why is the holistic approach becoming so important in landscape ecology?. Landscape and Urban Planning 2000; 50(1): 27-41.

[4] Naveh Z. What is holistic landscape ecology? A conceptual introduction. Landscape \& Urban Planning 2000; 50(1): 7-26.

[5] Forman RTT. Land Mosaics: The Ecology of Landscapes and Regions. Cambridge: Cambridge University Press; 1995.

[6] Fabbri P. Paesaggio, Pianificazione, Sostenibilità. Firenze: Alinea; 2003. See also by the same author, Principi Ecologici per la Progettazione del Paesaggio. Milano: Franco Angeli; 2007.

[7] Finotto F. Landscape assessment: the ecological profile. In: Cassatella C, Peano A, editors. Landscape Indicators: Assessing and Monitoring Landscape Equality. Dordrecht: Springer; 2011, p. 47-75.

[8] National Geographic website, http://education.nationalgeographic.org/encyclopedia/geographic-information-system-gis/ [accessed 14.10.2016].

[9] Turner MG. Landscape Ecology: the effect of pattern on process. Annual Review of Ecology and Systematics 1989; 20: 171-197.

[10] Pelorosso R, Della Chiesa S, Tappeiner U, Leone A, Rocchini D. Stability analysis for defining management strategies in abandoned mountain landscapes of the Mediterranean basin. Landscape \& Urban Planning 2011, 103(3): 335-346. 
[11] Pettit C, Cartwright W, Bishop I, Lowell K, Pullar D, Duncan D, editors. Landscape Analysis and Visualisation: Spatial Models for Natural Resource Management and Planning. Berlin: Springer-Verlag; 2008.

[12] Vermaat JE, Eppink F, van den Bergh JC, Barendregt A, Van Belle J. Aggregation and the matching of scales in spatial economics and landscape ecology: empirical evidence and prospects for integration. Ecological Economics 2005; 52(2): 229-237.

[13] Gobattoni F, Lauro G, Leone A, Monaco R, Pelorosso R. A procedure for the mathematical analysis of landscape evolution and scenarios assessment. Landscape \& Urban Planning 2011; 103(3): 289-302.

[14] Gobattoni F, Lauro G, Monaco R, Pelorosso R. Mathematical models in landscape ecology: stability analysis and numerical tests. Acta Applicandae Mathematicae 2013; 125: $173-192$.

[15] Boccaletti S, Latora V, Moreno Y, Chavez M, Hwang DU. Complex networks: structure and dynamics. Physics reports 2006; 424(4): 175-308.

[16] De Palma I. Methodologies of Landscape Ecology and Mathematical Models for Environments Evaluation and Ecoservice Systems Estimate. Master Degree Thesis, Politecnico di Torino; 2014.

[17] Smith HL. Monotone Dynamical Systems: an Introduction to the Theory of Competitive and Cooperative Systems. No. 41. American Mathematical Soc; 2008.

[18] Arnol'd VI. Catastrophe Theory. Berlin: Springer Science \& Business Media; 2003.

[19] Hirsch MW, Smith H. Monotone dynamical systems. Handbook of differential equations: ordinary differential equations 2006; 2: 239-357.

[20] Wilkinson JH. The Algebraic Eigenvalue Problem. Volume 87. Oxford: Clarendon Press; 1965. 